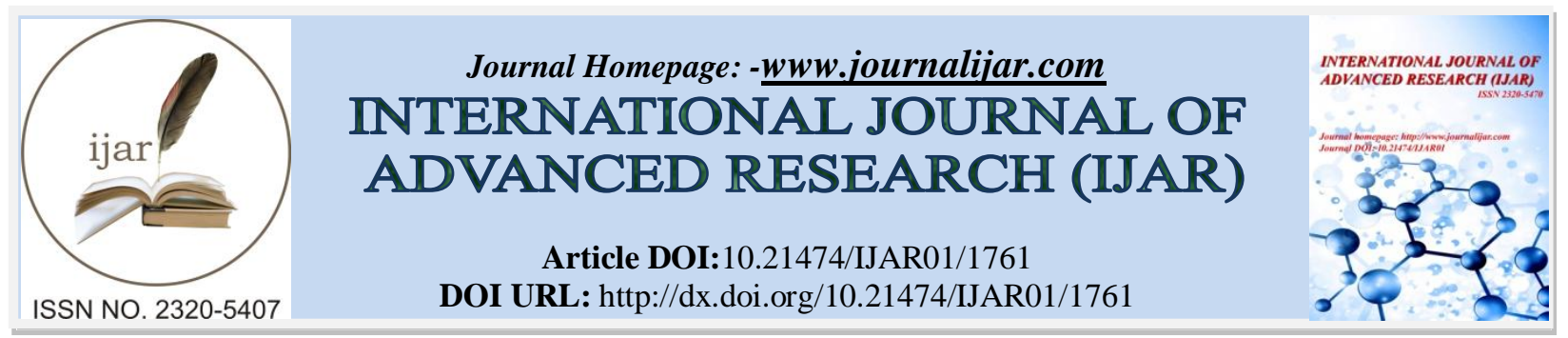

RESEARCH ARTICLE

\title{
SQUALENE IMMUNOGENICITY DETERMINATION AS AN ADJUVANT
}

Maha G. Soliman, Fatma A. Eid and AlyaM. Aly.

Department of Zoology, Faculty of Science, Al- AzharUniversity, Cairo, Egypt.

\section{Manuscript Info}

Manuscript History

Received: 12 August 2016

Final Accepted: 22 September 2016

Published: October 2016

Key words:-

Squalene

Cytokines

Inflammation

Lymph node

Histopathology

\begin{abstract}
The concept that vaccines prevent the spread of infectious disease is based upon the belief that, by injecting a small amount of a disease into the body, it will develop "antibodies" that will prevent the injected person from contracting the disease against which he had been vaccinated. The fact that attenuated doses of pathogens alone will not initiate an "antigenic response"; vaccines must contain compounds known as "adjuvants" to intensify the body's immune response. Squalenehas been added to vaccine asimmunologic adjuvant to stimulate the immune system, increase the response to the vaccine and reduce amount of usage vaccine. The objective of this study is to investigate the possible immunological effects of squalene as an adjuvant, Albino rats were injected with two doses of squalene (AS03) at interval three weeks between them. Results obtained in the present study showed that squalene as adjuvant contributed to the initiation of the innate immune response, exemplified by cytokines elevation, inflammatory responses and activated effectors that move to draining lymph nodes and activating the adaptive immune response. This study provides evidence that squalene is responsible for the regiment of adverse reactions, which act as detergents instead of its helper role of vaccine. Based on the results presented here, it may be concluded that squalene has a toxicity effectors in the biological pathways including, immunopathological and histopathological effects. These could be related toa strong immunostimulation effect of squalenewhen it potentiated the vaccine as immunoenhancers.
\end{abstract}

Copy Right, IJAR, 2016,. All rights reserved.

\section{Introduction:-}

The theory of stimulating the body's immune response is the basis underlying vaccination. Vaccines act by initiating the innate immune response and activating antigen presenting cells, thereby inducing a protective adaptive immune response. Adjuvants are substances added to vaccines to enhance the immunogenicity of highly purified antigens that have insufficientimmunostimulatory capabilities.Ithad been used in human vaccines for more than 90 years. While early adjuvant oil-in-water emulsions (squalene) was used empirically, rapidly increasing knowledge on how the immune system interacts with pathogens means that there is increased understanding of the role of adjuvants and how the formulation of modern vaccines can be better tailored towards the desired clinical benefit (Alberta $e t$ al., 2015). Adjuvants are often included in vaccines to enhance the recipient's immune response to a supplied antigen while keeping the injected foreign material at a minimum dose (David and Andrew, 2009). Pandemic-influenza vaccines containing split-inactivated-virus antigen have been formulated with the immunostimulatory adjuvant

Address:-Department of zoology, faculty of science, al- azhar university, cairo, Egypt. 
systemAS03. The administration of repeated doses of the AS03 vaccine was primarily associated with transient mild inflammation at the injection site and draining lymph nodes. The biodistributionkinetics of AS03 constituents were consistent with AS03 inducing this pattern of inflammation(Segalet al., 2015). The pathologic reaction to the adjuvants starts at the injection site with mild erythema, muscles aches and swelling (Ho, 2009b), followed by tissue necrosis, intense inflammation and granulomatous lesion (Viera, 2001). Adjuvants can break tolerance meaning and they can disable the immune system to the degree that it loses its ability to distinguish what is 'self' from what is foreign. Normally, the immune system ignores the constituents of one's own body, but if something happens to break tolerance, the immune system turns relentlessly self-destructive and attacking the body (Jeff, 2015). According to Asaet al. (2000)the damage is done by vaccines, particularly when potentiated by powerful "immunoenhancers". It is a potent Th1-response-inducing adjuvant when formulated in a squalene oil-in-water emulsion (Anthony et al., 2015). Barbroet al. (2000)reported that a single injection of the squalene as adjuvant into rats triggered "chronic, immune-mediated joint-specific inflammation" also known as rheumatoid arthritis. Dueto many pathologic changes that occurred after squalene immunization, the present study was planned to determine the immune response and the histopathological changes in the lymph nodes of Albino rats.

\section{Materials and methods:- Animals:-}

Young Albino rats weighing 65-70 gm were obtained from The Nile Company for Pharmaceutical and Chemical Industries. After an acclimatization period of one week, the animals were housed in bottomed cages in a room under standard conditions. They were provided with water and a balanced diet. All animals received care in compliance with the Egyptian rules for animal protection.

\section{Study design:-}

Animals were divided into different treatment groups as follows: the first group (Con), the second group received intramuscular injection of squalene adjuvant $(\mathrm{Sq} 1, \mathrm{AS} 03)$ at the zero day and the third group received second intramuscular injection dose at the $21^{\text {st }}$ day (Sq2, AS03). Animals of all the treated groups vaccinated with $0.125 \mathrm{ml}$, the same dose of a human multiplied by conversation factor (0.018)/200 gm of body weight of rat according to Paget and Barnes (1964). Animals of all groups were decapitated after 10 and 30 days post-treatment $(n=10)$. The collected serum was assayed for immunological parameters.Lymph nodes of rats were carefully removed and preparedto use for the various histopathological and histochemical determinations.

\section{Immunological Studies: Cytokine Assays:-}

Serum were harvested after 10-(Sq1) and 30-(Sq2) days post-immunizationin order to estimate interferon gamma(IFN- $\gamma$ ) according to Kelder and Rashaidbaigi (1986), interleukin-10 (IL-10) according to Everson (2005) and tumor necrosis factor(TNF- $\alpha$ ) according to Brouckaert (1993) using a sandwich enzyme-linked immunosorbent assay (ELISA).The resulting optical density was read on a microtitre plate reader (ELX-808, BioTek Instruments, Winooski, VT, USA) with $450 \mathrm{~nm}$ wavelength correction.

\section{Histological examinations of the lymph node:-}

Lymph nodes of immunized rats were washed in saline and fixed in $10 \%$ neutral formalin, embedded in paraffin, sectioned at $5 \mu \mathrm{m}$ and stained with haematoxylin and eosin stain according to the method of Drury and Wallington (1980) and Mallory`s trichrome stain according to the method of Pearse(1977).

\section{Histochemical examinations of the lymph node:-}

Lymph node tissues of immunized rats were prepared and stained with periodic acid Schiff technique according to Pearse (1977) and mercuric bromophenol blue according to the method of Maziaet al.(1953) for light microscopic observations.

\section{Statistical analysis:-}

Results were expressed as the mean \pm standard error of the mean (SEM). Data for multiple variable comparisons were analyzed by one-way analysis of variance (ANOVA). For the comparison of significance between groups a post hoc testwas used according to the statistical package program (SPSS version 17.0). 


\section{Results:-}

Immunological changes of cytokines:-

Interferon - gamma (INF - $\gamma)$ :-

Results of Fig. 1 summarize the effect of squalene immunization that produced disturbances in interferon- gamma level. In view of the present results, stimulation with squalene as adjuvant exhibited a significant $(p<0.05)$ increase in the INF $-\gamma$ level after the first (Sq1) and the second (Sq2) dose compared to the control group.

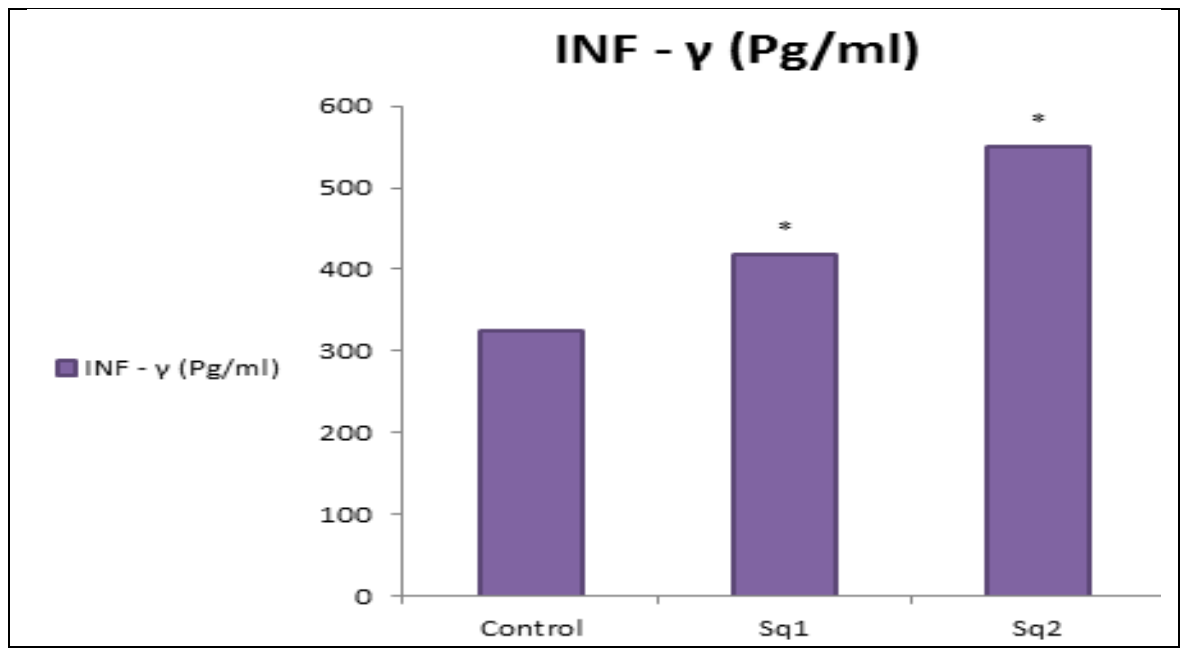

Fig 1:- Comparative evaluation of interferon- $\gamma$ level realized from squalene- immunized groups after the first (Sq1) and the second ( $\mathrm{Sq} 2)$ dose to non-immunized control group. (* $\mathrm{p}<0.05$ vs. control group).

\section{Interlukin-10 (Rat- IL-10):-}

As shown in Fig. 2 immunization of squalene on given for zero and 21 days interval were produced elevation in interleukin-10level. Stimulation with squalene as adjuvant exhibited a significant increase in the IL- 10 compared to the control group after the first (Sq1) and the second (Sq2) dose of immunization. Deposit the fact that it recorded higher values in squalene- immunized group after the second (Sq2) than squalene- immunized group after the first (Sq1) dose.

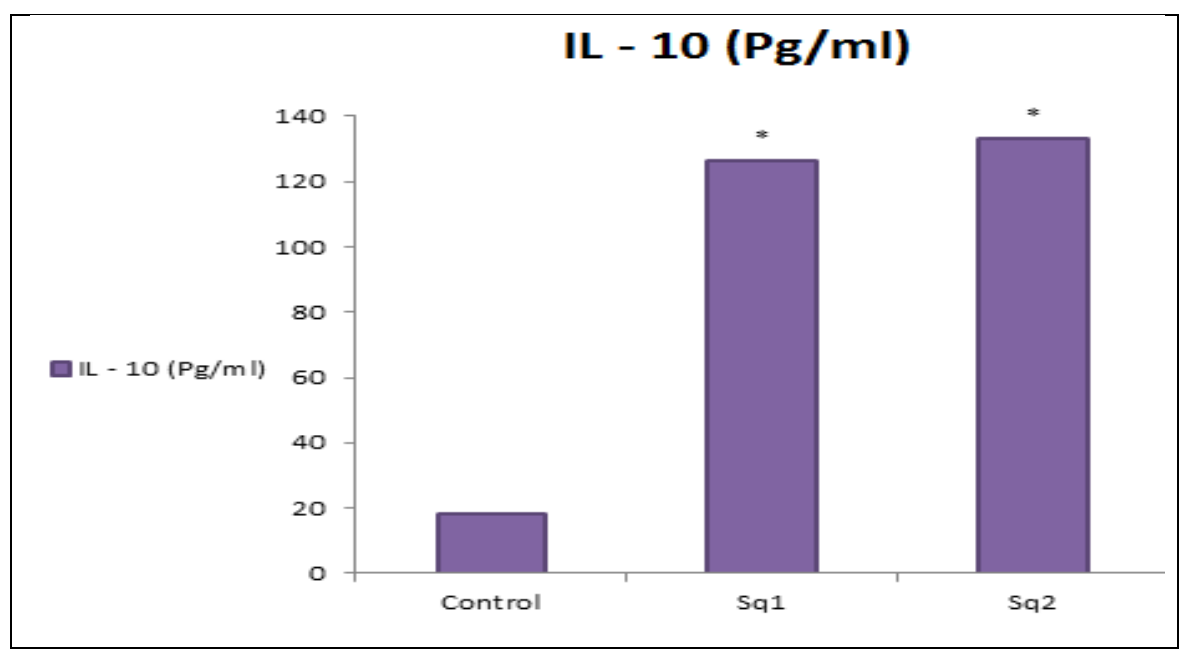

Fig 2:- Comparative evaluation of interleukin-10 level realized from squalene- immunized groups after the first (Sq1) and the second (Sq2) doseto non-immunized control group. (* $\mathrm{p}<0.05$ vs. control group). 


\section{Tumor necrosis factor(TNF)-alpha:-}

In Fig. 3a significant increase $(\mathrm{p}<0.05)$ in TNF $-\alpha$ was observed all over the experimental periods of squaleneimmunized groups after the first (Sq1) and the second (Sq2) dose compared to the control group.

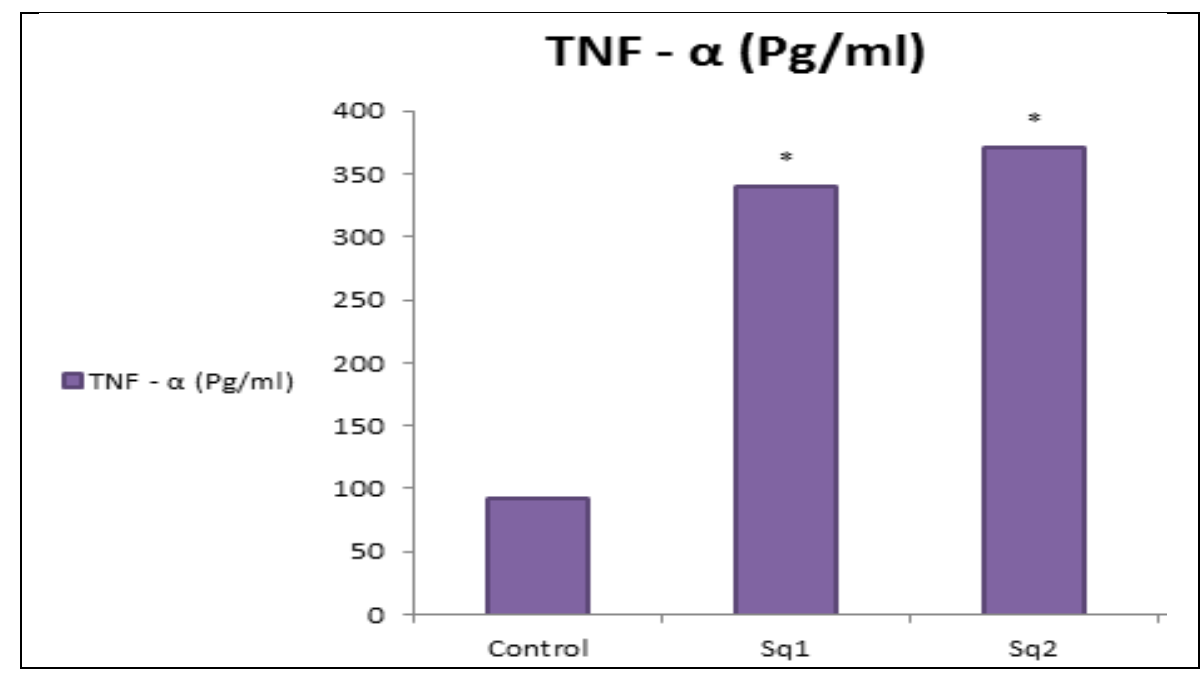

Fig 3:- Comparative evaluation of tumor necrosis factor (TNF) - $\alpha$ level realized from squalene- immunized groups after the first (Sq1) and the second (Sq2) dose to non-immunized control group. (* p $<0.05$ vs. control group).

\section{Histopathological changes in rat lymph node tissue:-}

Representative sections of the lymph node are shown in Fig. 4A-C and Fig. 5A-C. Lymph node tissue sections from a rat of the control group stained with hematoxylin and eosin showed normal histological features capsule, cortex, cortical follicle, germinal center, paracortex and medulla with medullary cords (Fig. 4A). Sections of squalene treated group after the first dose (Sq1) showing that lymph node lost its normal architecture, numerous degenerated areas, dialated and congested blood sinus. Elongated medulary cords, fibrosis with bizarre arrangement of lymphocytes in the cortex and medulla, deeply stained nuclei in numerous lymphocytes (pyknosis) and rupture of the capsule are also noticed. Also, lymph node showed adisappearance of the cortical follicles and medullary cords with numerous hemorrhagic areas (Fig. 4B). Otherwisesqualene treated group after the second dose (Sq2) showed numerous of degenerated areas especially in the medulla, dialated and congested cortical and medullary sinus, elongated medulary cords and large hemorrhagic areas inside and outside the lymph node.In addition lymph node lost its normal architecture that's contained delaminated and distorted capsule, diffused stain affinity of lymphocytes in the cortical follicle and numerous deeply stained nuclei (pyknotic) especially in the helium region (Fig. 4C).

The rat lymph node tissue of the control group at the present study that stained with Mallory's trichrome stain showed normal histological features with dense stain affinity of collagen fibers in the capsule and scattered bundles in the cortex and medulla especially around the blood sinuses (Fig. 5A). Highly increased collagen fibers in and around the capsule, cortex and in the medulla especially near the helium and in the degenerated areas were also observed in squalene treated group after the first dose (Sq1) (Fig. 5B). Sections of squalene treated group after the second dose (Sq2) showed nearly occupied lymph node tissue with collagen fibers. Notice: large hemorrhagic areas which acquired brightly red color (Fig. 5C). 

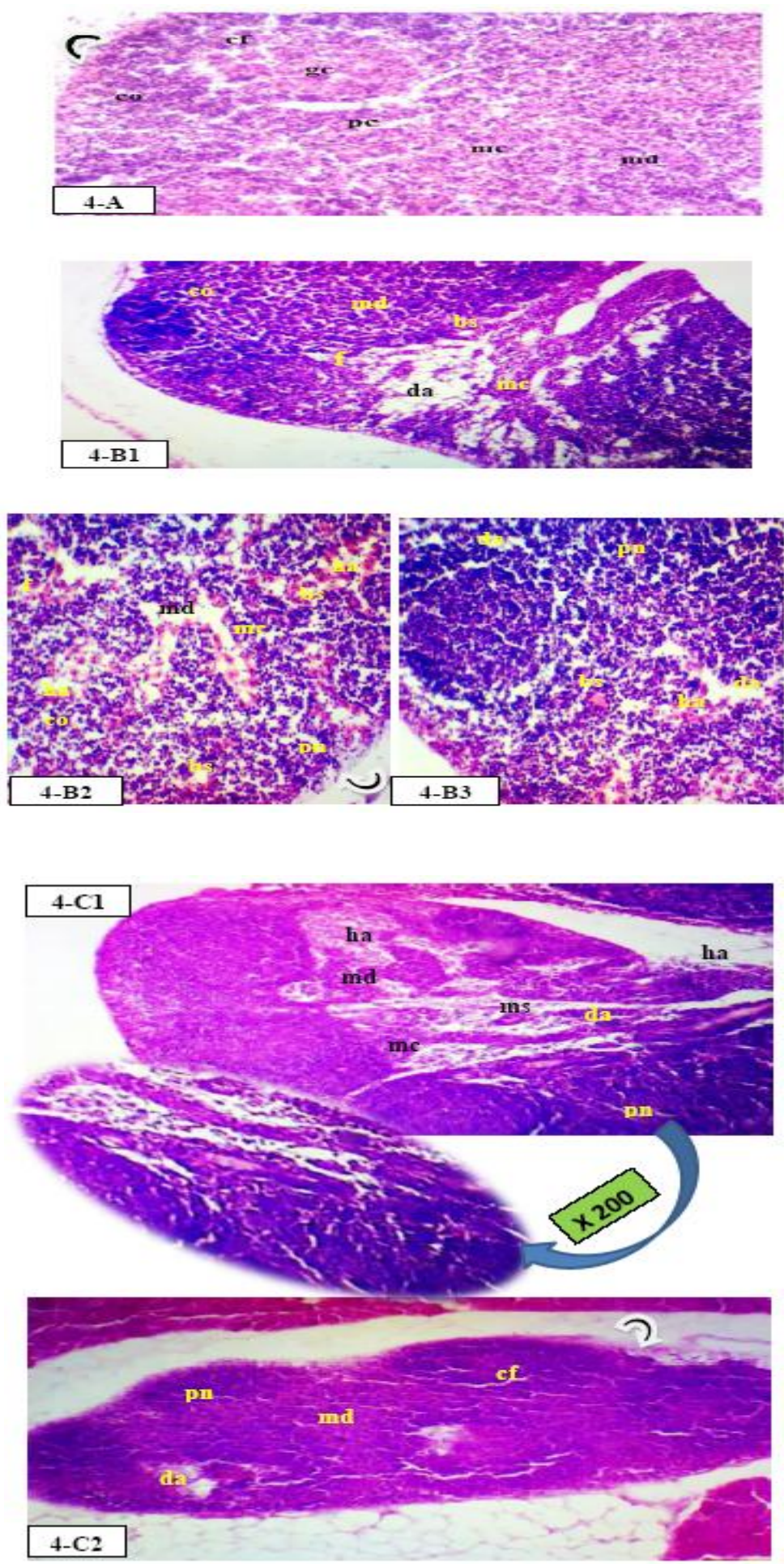

Fig 4:- Representative images of histopathological changes in rat lymph node tissue (H\&E, original magnification x 100,B2, B3x 200) (A-C), ( $=10$ rats in each group). (A) Control group: $\uparrow-$ capsule, co -cortex, cf -cortical follicle, gc germinal center, pc-paracortex, md -medulla, mc medullary cords. (B1, 2, 3)Squalene treated group after the first dose (Sq1): da -degenerated areas, bs - blood sinuses, mc -medulary cords, $f$-fibrosis, co -cortex, md -medulla, pn -pyknosis, $\uparrow$-capsule, Ha-hemorrhagic areas. $(\mathbf{C 1}, 2)$ Squalene treated group after the second dose (Sq2): da- degenerated areas, md -medulla, ms- medullary sinus, mc- medulary cords, ha- hemorrhagic areas, $\uparrow$ capsule, cf -cortical follicle, pn -pyknotic, h- helium region. 

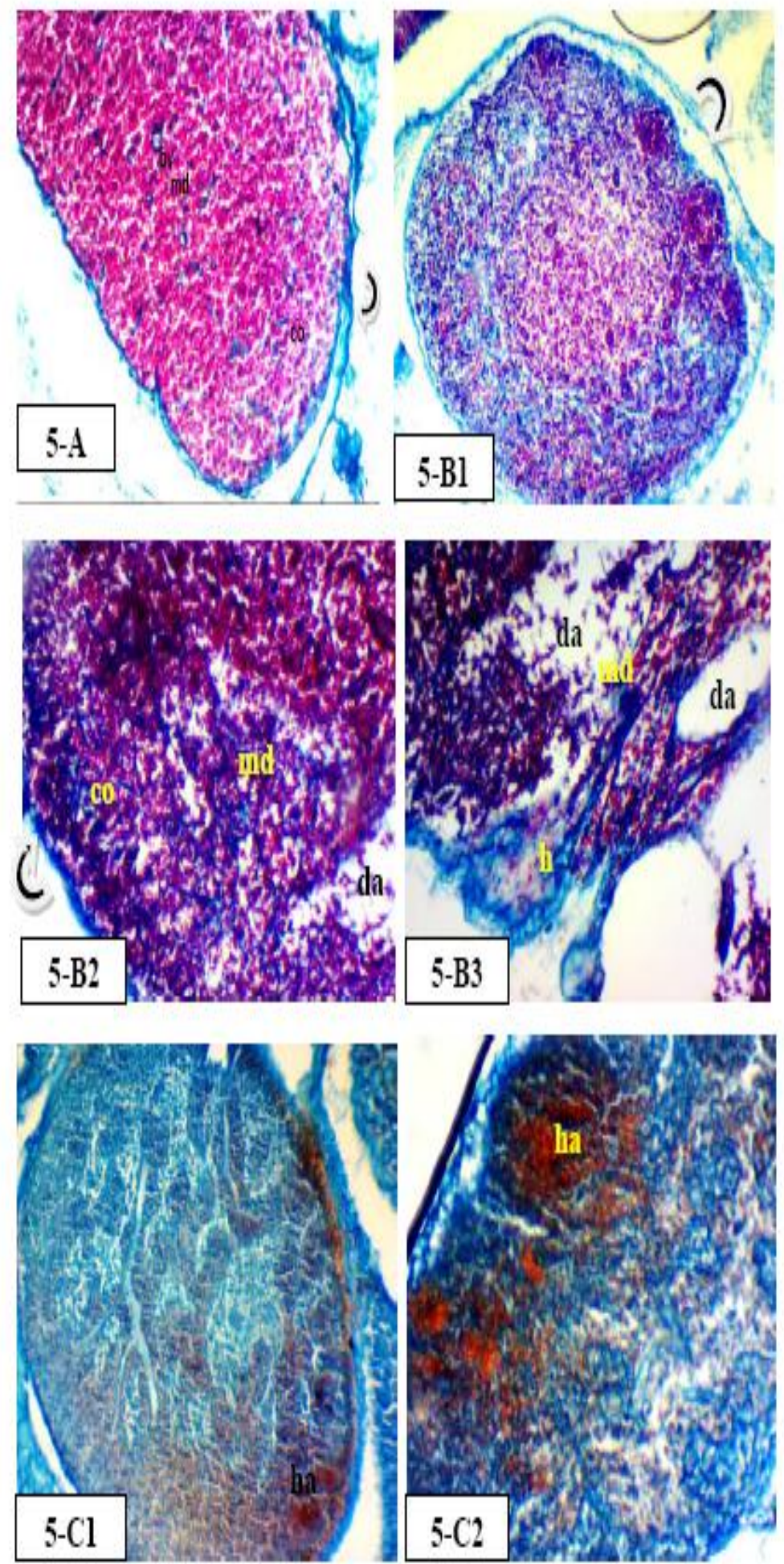

Fig 5:- Representative images of histopathological changes in rat s lymph node tissue (Mallory's trichrome stain, original magnification $\mathrm{x} 100)(\mathrm{A}-\mathrm{C}),(\mathrm{n}=10$ rats in each group). (A) Control group: $\uparrow$ - capsule, co- the cortex, mdmedulla, bs- blood sinuses. (B1, 2, 3) sections of squalene treated group after the first dose (Sq1): $\uparrow$ - capsule, cocortex, md- medulla, h- helium, da- degenerated areas. $(\mathbf{C 1}, 2)$ sections of squalene treated group after the second dose (Sq2): ha- hemorrhagic areas. 


\section{Histochemical changes in rat lymph node tissue:-}

Results of histochemical changes in the lymph node are shown in Fig. 6A-C and Fig. 7A-C. Lymph node tissue of rats of the control group stained with periodic acid Schiff's reaction (PAS) showed normal distribution of PAS +ve materials, notice: remarkable staining affinity in the capsule and walls of the blood sinuses (Fig. 6A), lymph node tissue of the squalene treated group after the first dose (Sq1) showed poorly stained lymphocytes in some cortical areas and medulla, but other cortical follicles, the capsule, walls of the dilated blood sinuses and trabeculae are deeply stained (Fig. 6 B). Our results further indicate that after the second dose (Sq2) treatment reduction of stain affinity of PAS +ve materials in the cortex and medulla were observed, but degenerated areas were negatively stained. Notice: thick walls of the blood sinuses (bs) were deeply stained (Fig. 6 C). Lymph node tissue of rats of the control group stained with mercuric bromophenol blue showed normal distribution of total protein in the lymph node tissue especially in the cortex and medulla, while dense staining affinity in the capsule, cortical follicle and walls of the blood sinuses were also noticed (Fig. 7A). Highly reduced staining affinity of total protein in the lymph node tissue with deeply stained capsule and negatively stained degenerated areas are noticed in squalene treated group after the first dose (Sq1) (Fig. 7B) and squalene treated group after the second dose (Sq2) (Fig. 7C).
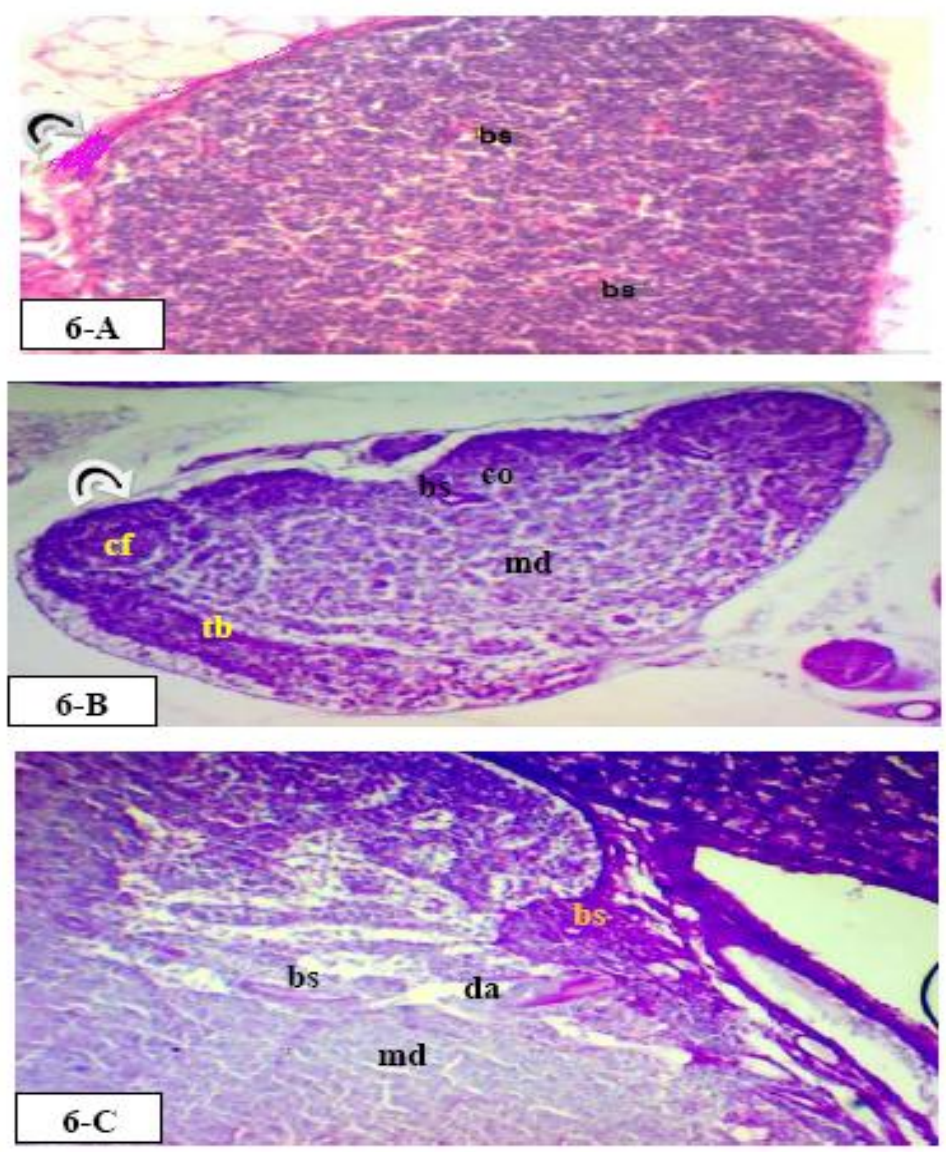

Fig 6:- Representative images of histochemical changes in rat lymph node tissue (periodic acid Schiff's reagent (PAS), original magnification $\mathrm{x} 100)(\mathrm{A}-\mathrm{C}),(\mathrm{n}=10$ rats in each group). (A) Control group: $\uparrow$-capsule, bs- blood sinuses. (B)Squalene treated group after the first dose (Sq1): co- cortical areas, md- medulla, cf- cortical follicles, $\uparrow$ - the capsule, bs- blood sinuses, tb- trabeculae. $(\mathbf{C 1}, 2)$ Squalene treated group after the second dose (Sq2): cocortex, md- medulla, da- degenerated areas, bs- blood sinuses. 

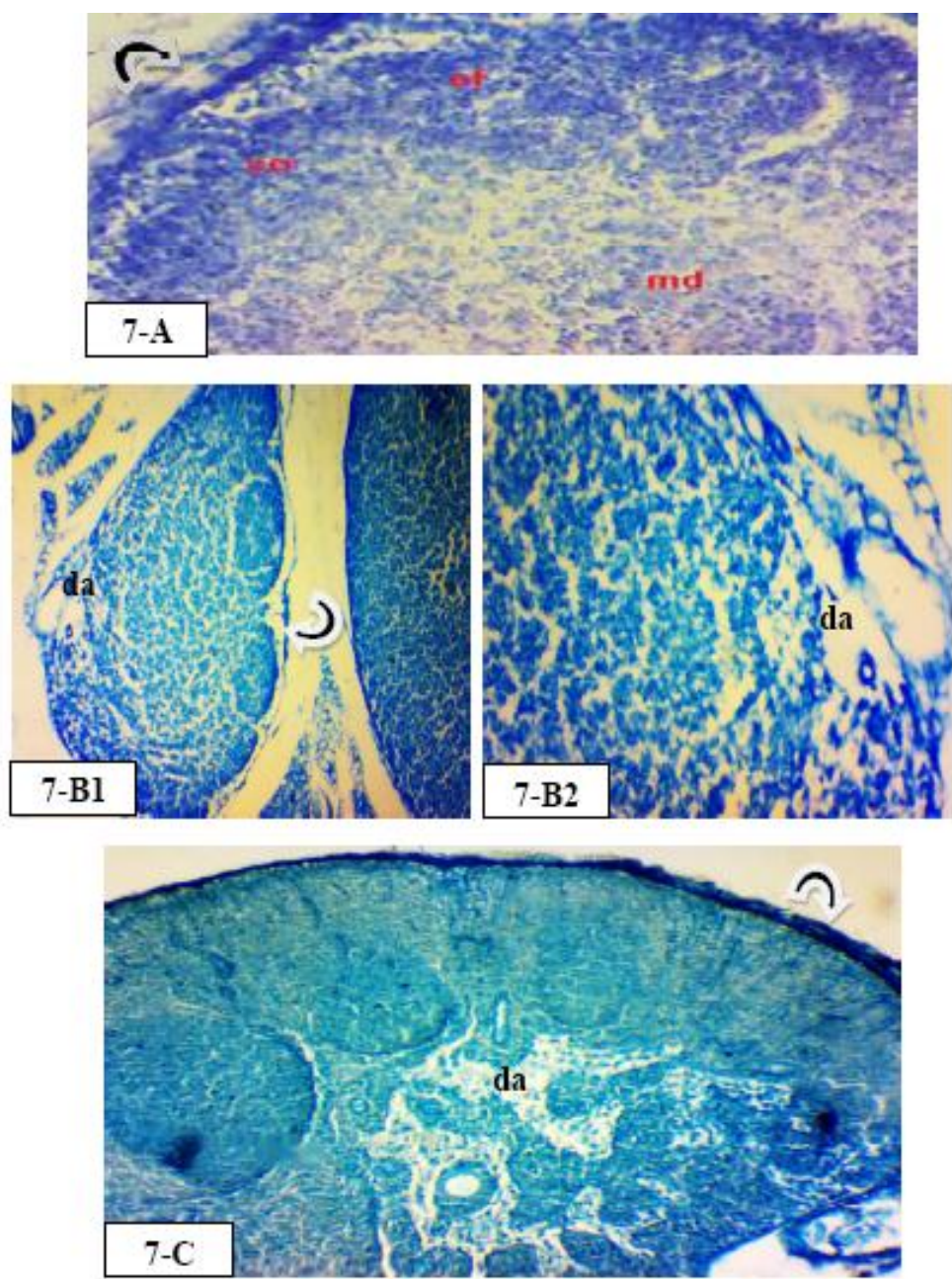

Fig 7:- Representative images of histochemical changes in rat lymph node tissue (mercuric bromophenol blue, original magnification x 100) (A-C), ( $\mathrm{n}=10$ rats in each group). (A) Control group: co- cortex, md- medulla, $\uparrow-$ capsule, cf- cortical follicle, bs- blood sinuses. (B1, 2)Squalene treated group after the first dose (Sq1): $\uparrow$ - capsule, da- degenerated areas. (C)Squalene treated group after the second dose (Sq2): $\uparrow$ - capsule, da- degenerated areas.

\section{Discussion:-}

Many of the immunological changes appeared in the present study as significantly increased differences at 10 and 30 days post-immunization compared with control group,this result is in agreement with those of Calabroet al.(2013)who stated that squalene oil-in-water emulsions elicit both cellular (Th1) and humoral (Th2) immune responses, this class of adjuvants is believed to act through stimulation of cytokines and chemokines production by macrophages and granulocytes. This investigations were explained byDidierlaurentet al. (2014) who declared that the requirement of an adjuvant-induced inflammatory milieu was elegantly demonstrated in mice, where sequential delivery of the adjuvant followed by protein antigenswere necessary to obtain antigen-specific immunity, thus the adjuvantdeliveryseems capable of initiating inflammation to promote infiltration of circulating immune cells (Calabroet al., 2013). Indeed, chemokines and cytokines were readily detected in mouse injected with distinct adjuvants as early as $3 \mathrm{~h}$ post injection, in addition increased numbers of neutrophils have been found for up to $48 \mathrm{~h}$ in adjuvant-injected mouse( $\mathrm{Lu}$ and Hogenesch, 2013). Also these results are in line with those of Monteiroet al. (1998) who stated that the adjuvanted vaccine has been shown to contribute to proliferation of IFN- $\gamma$ production and a cytolytic response after vaccination. Therefore, the high concentrations of IFN- $\gamma$ cytokines suggest that adjuvanted vaccine was probably more effective in stimulation of naive CD8 cells and thus a cytotoxic response than influenza infection (Huckriedeet al., 2005). The highly elevation of IFN- $\gamma$ recorded in the present work after the second dose than the first one agrees with the results of Szyszkoet al. (2006) who emphasized that the virus vaccine induced a significantly higher IFN- $\gamma$ after the first and the second vaccinations in mice. In 
additionKosalaraksaet al. (2015)stated that immunogenicity against AS03-adjuvanted vaccine and placebocontrolled strain was assessed 21 days after the first and second vaccine doses for all vaccines, the adjuvanted vaccine was more reactogenic than placebo. All of these findings may be due to immunostimulation action of squalene as adjuvant, this investigation in agreement with those of Szyszkoet al. (2006) who had shown that adjuvantedvaccine when prepared from inactivated virus it causes immunostimulation effect. The results of current study showed elevation of IL-10 values that produced by squalene immunization all over the experimental periods. Szyszkoet al.(2006)also reported that influenza vaccine induced a strong mixed cytokine response particularly in induction of Th2 of cytokines especially after the second dose and substantially influences the magnitude and quality of the immune response in addition to the expression of type 2 cytokine. Rather than Karen et al. (2013) recorded that splenocytes from mice received intramuscular injection of influenza A/ vaccine formulated with AS03 produced higher levels of IL-10 than those from mice immunized with antigen alone. This adjuvant stimulates leukocyte recruitment, antigen transport to drain lymph nodes and stimulate lymphocyte priming (Seubertet al., 2011). In the present work a higher significant increase of TNF- $\alpha$ produced by squalene stimulation were realized at different intervals (21 and 42 days post-stimulation), these results were accepted by Liang et al. (2015)who stated thatinduction of an efficient vaccine response requires some degrees of local inflammation to trigger and support the sequence of immunological events leading to the adaptive immunity. Vaccine adjuvants administered to the muscle have a central role in inducing transient inflammation at the delivery site that promotes immune cell recruitment and activation, this inflammation likely leads to better vaccine antigen uptake by critical infiltrating cell types and migration of vaccine-loaded cells to the draining lymph nodes (dLNs) to establish the adaptive immunity. In addition Szyszkoet al. (2006) stated that all the four inflamatory cytokines (GM-CSF, IL-1 $\beta$, IL-6 and TNF- $\alpha$ ) were elevated in vaccinated mice. Generally, the vaccine caused autoimmunity and inflammatory diseases may be due to over immunostimulation and inflammatory action of squalene as adjuvant that demonstrated by high elevation of TNF- $\alpha$, this suggestion comes in the same line with those of Eidet al. (2015). These autoimmunity and inflammatory effects of the adjuvanted vaccine are correlated to many inflammatory diseases such as arthritis, granulomatous lesion, atherosclerosis, inflammatory bowel disease (Mukhopadhyayet al., 2012) and other diseases that accompanied by macrophage elevation (Ho, 2009a).Harold (2005) stated that the adjuvant causes a variety of proinflammatory cytokines including interleukin (IL-10), tumor necrosis factor (TNF), IL-6, IL-12 and CXCL8 (IL8). The author added that the secretion of IL-12 by dendritic cells (DC) generate a TH1 response through IFN- $\gamma$ induction in $\mathrm{T}$ cells. As well as the adjuvantcauses immune-stimulating complexes (ISCOMs1) (Gomez-Vargas et al., 2004).

Lymph node tissues of the present study showed many drastic changes of the enlarged lymph node that lost its normal architecture in squalene - treated group after the first dose,this result in agreement with those of Ann et al. (2012) who found that morphologic changes occurring in germinal centers and paracortical follicles of draining lymph nodes at two intervals after sensitization of guinea pigs with adjuvant. Numerous pyknotic nuclei were observed in cells of the lymph node tissue in the present study after the second dose,which is in agreement with the opinion ofEidet al. (2015). Otherwise Viera (2001)who found that the adjuvant induced cytotoxic T-lymphocyte (CTL), some types were found in mesenteric lymph nodes and in the spleen. All of these notifications may be due to squalene as adjuvant and this suggestion is in agreement with those of Viera (2001) who documented that the pathologic reaction to the adjuvants starts at the injection site with mild erythema and swelling followed by tissue necrosis, intense inflammation and the usual progression to the formation of a granulomatous lesion. This also may clear the presence of fibrosis in squalene - treated groups, where granuloma is a medical term for collection of macrophages(Mukhopadhyayet al. 2012).Highly elevated collagen fibers were realized in the present study in addition to nearly occupied lymph node tissue with collagen fibers in squalene - treated group after the second dose was also realized by Kandeal (2016) under different factors. All of these investigations may be due to the detrimental effects on the tissue, granulomatous lesion and lymphoma formation as overstimulation of immune responses. This was mentioned by Hirano (2012) who found that injection of Bordetella pertussis vaccine augmented growth of a lymphoma cell that causes cell death. Reduced stain affinity of total protein may be due to damaged protein molecules by detrimental and toxic action of vaccine components such as squalene this suggestion comes in the same line with those of Eidet al. (2015). This finding is in accordance with those of Kapyahoet al. (1983) who reported that decreased protein synthesis could be attributed to degeneration of cellular tissues. In addition to vacoulation and degeneration caused by tissue toxicity of squalene in this study which is responsible for decreased protein content in the lymph node tissue, these suggestion comes in the same line with those of Abdel Rahmanet al. (2013)who found that sections of squalene as adjuvant, exposed brains showed vacuolation and degenerated neurons as well as necrotic and apoptotic neurons in hypothalamus, striatum and cerebral cortex. 
According to Khaki et al. (2006) decreased polysaccharides content in the present study may be related to lymphoma activation and fibrosis.

\section{Conclusions:-}

From the presented data it could be concluded that squalene as adjuvant that used in many vaccines showed a higher immune potential after two doses than one only. Despite the adverse events induced post administration of squalene that showed slowly recovery after long period effect. Squalene as adjuvant showed a drastic immunoological changes in addition to the sever immunohistological and histopathological changes.

\section{Acknowledgement:-}

The authors are thankful to Dr. Aly F. Mohamed, Prof. of Virology, Applied research manager, The Holding Company for Biological Products and Vaccines (VACSERA) andDepartment of Zoology, Faculty of Science (Girls branch), Al- Azhar Universityfor providing laboratory facilities and technical assistance.

\section{References:-}

1. Abdel- Rahman, M., Mohamed, A., Essam, N. andAbdel - Moneim, A. (2013): Studies on H1N1 vaccineinduced monoamines alternations and oxidative stress on brain of adult mice. J. Appl. Pha. Sci., 3(04): 48-53.

2. Alberta, D., Scott, P., Fernanda, T. and Nathalie, G. (2015): Vaccine Adjuvants: from 1920 to 2015 and Beyond.Vaccines, 3: 320-343.

3. Ann, D., Robert, B. and Harold, D.(2012):Morphologic changes in draining lymph nodes and in lymphocyte cultures after sensitization with complete or incomplete Freund's adjuvant.J. Iттио., 188(8): 422-435.

4. Anthony, L., Desbien, J., Reed1, R., Bailor1, Natasha, C., John, L., Mark, O., Christopher, F.,Darrick, C., Steven, G. and Malcolm, D. (2015): Squalene emulsion potentiates the adjuvant activity of the TLR4 agonist, GLA, via inflammatory caspases, IL-18 and IFN- $\gamma$. European Journal of Immunology,45(2): 407-417.

5. Asa, B., Cao, Y. and Garry, F. (2000): Antibodies to squalene in Gulf War Syndrome. Exp. Mol. Pathol., 68(1): $55-64$.

6. Barbro, C., Asa, J., Anders, L., Anders, B. and Johnny,L. (2000): The endogenous adjuvant squalene can induce a chronic T-cell-mediated arthritis in rats. American Journal of Pathology, 156 (6): 2057-2065.

7. Brouckaert, P. (1993): Tumor necrosis factor, its receptors and theconnection with interleukin 1 and interleukin 6. Immunobiology, 187:317-29.

8. Calabro, S., Tritto, E., Pezzotti, A., Taccone, M., Muzzi, A. andBertholet, S. (2013): The adjuvant effect of MF59 is due to the oil-in-water emulsion formulation, none of the individual components induce a comparable adjuvant effect. Vaccine, 31: 3363-3369.

9. David, F. and Andrew, P. (2009): What the heck is an adjuvant, anyway?. In: Swine Flu Vaccine. Joseph Brownstein, ABC News Medical Unit, http://abcnews.go.com/Health/SwineFluNews/story?id=8296948.

10. Didierlaurent, M., Collignon, C., Bourguignon, P., Wouters, S., Fierens, K. andFochesato, M.(2014): Enhancement of adaptive immunity by the human vaccine adjuvant AS01 depends on activated dendritic cells. J. Immunol., 193: 1920-1930.

11. Drury, R. and Wallington, E. (1980): Carleton's Histological Technique, $4^{\text {th }}$ Ed. Oxford, Univ. Press, New York, Toronto.

12. Eid, F., Mohamed, A., Aly, A. and Ibrahim, N. (2015): Effects of Swine flu (H1N1) vaccine on Albino rats. Journal of Bioscience and Applied Research, 1(3): 113-126.

13. Everson, C. A. (2005): Quantitative determination of interleukin-10 in rat serum, buffered solution and cell culture medium. Am. J. Regul. Intrgr. Comp. Physiol., 289(4): 1054-1063.

14. Gomez-Vargas, A., Rosenthal, K., McDermott, M. and Hortelano, G. (2004): Continuous antigenic stimulation system (CASS) as a new immunization strategy. Vaccine, 22:3902-3910.

15. Harold, S. (2005):Adjuvants and antibody production: dispelling the myths associated with Freund's complete and other adjuvants. ILAR Journal, 46(3): 280-293.

16. Hirano, M., Sinkovics, J., Shullenberger, C. and Howe, C. (2012): Murine lymphoma: augmented growth in mice with pertussis vaccine- induced lymphocytosis. WebTarg. Tumor Vuln., 158 (3804): 1061-1064.

17. Ho, M. (2009a): Fast-tracked swine flu vaccine under fire. Science in Society, 43: 4-6.

18. Ho, M. (2009b): Cardiovascular risks from swine flu vaccines. Science in Society, 43: 147-154.

19. Huckriede, A., Bungener, L. andStegmann, T. (2005): The virosome concept for influenza vaccines. Vaccine,23(1): 26-38. 
20. Jeff, R. (2015): Squalene - ahistory of vaccine development and the newest adjuvant, http://12160.info/profiles/blogs/squalene-a-history-of-vaccine

21. Kandeal, H. (2016): The possible radioprotective role of Aphanizomenonflos-aquae (AFA) on adult male albino rats. M.Sc. Thesis, Zoology Department, Faculty of Science, Al AzharUnivisity.

22. Kapyaho, K., Lauharanta, J. and Janne, J.(1983): Inhibition of DNA and protein synthesis in U.V. irradiated mouse skin by 2-difluoromethy lornithine, methylglyoxalbis (Guanylhydrazone) and their combination. $J$. Invest. Derm., 81: 102-106.

23. Karen, Y., Angela, B., Jyotsana, G., Elizabeth, A. andBrian, W. (2013):Long-term immune responses to AS03adjuvanted, low antigen dose influenza vaccines in BALB/c mice (P4299). The Journal of Immunology, 190: 123-133.

24. Khaki, A., Tubbs, R., Shoja, M., Rad, J., Khaki, A., Farahani, R., Zarrintan, S. and Nag, T.(2006): The effects of an electromagnetic fields on the boundary tissue of the seminiferous tubules of the rat: A light and transmission electron microscope study. Folia. Morphol., 65(3): 188-194.

25. Kelder, B. and Rashidbaigi, A. (1986): A Sandwich radioimmunoassay for human IFN- $\gamma$. In: Methods in Enzymology. Vol. 119, Academic Press, New York, pp. 582-587.

26. Kosalaraksa, P., Jeanfreau, R., Frenette, L., Drame, M., Madariaga, M., Innis, L., Godeaux, O., Izurieta, P. andVaughn, W. (2015): AS03B-adjuvanted H5N1 influenza vaccine in children 6 months through 17 years of age: a phase 2/3 randomized, placebo-controlled, observer-blinded trial. J. Infect. Dis., 211(5): 801-810.

27. Liang, F., Ploquin, A., Hernandez, D., Fausther-Bovendo, H., Lindgren, G. and Stanley, D. (2015): Dissociation of skeletal muscle for flow cytometric characterization of immune cells in macaques. J. Immunol. Methods, 425:69-78.

28. Lu, F. andHogenesch, H. (2013): Kinetics of the inflammatory response following intramuscular injection of aluminum adjuvant. Vaccine, 3: 3979-3986.

29. Mazia, D., Brewer, P. andAlfert, M. (1953): The cytochemical staining and measurement of protein with mercuric bromophenol blue. Biol. Bull., 104: 57 - 67.

30. Monteiro, J., Harvey, C. and Trinchieri, G.(1998): Role of interleukin-12 in primary influenza virus infection. J. Virol., 72: 4825-4831.

31. Moustafa, N. and Hafez, M.(1998): The possible protective effects of Nigella sativa on mice fetuses of mothers exposed to $\gamma$-rays. Egypt J. Histol., 21(2): 235-250.

32. Mukhopadhyay, S., Farver C., Vaszar, L., Dempsey, O., Popper, H., Mani, H., Capelozzi, V., Fukuoka, J., Kerr, K., Zeren, E., Iyer, V., Tanaka, T., Narde, I., Nomikos, A., Gumurdulu, D., Arava, S., Zander, D. and Tazelaar, H.(2012): Causes of pulmonary granulomas: a retrospective study of 500 cases from seven countries. J. Clin. Pathol., 65 (1): 51-57.

33. Paget, G. E. and Barnes, J. M.(1964): Interspecies dosage conversion scheme in evaluation of results and quantitative application in different species. Evaluation of Drug Activities: Pharmacometric, 1: 160-162.

34. Pearse, A.(1977):Histochemistry, Theoretical, and Applied. $3^{\text {rd }}$ ed., vol. 1. Churchill Livingstone, London.

35. Segal, L., Wouters, S., Morelle, D., Gautier, G., Le Gal, J., Martin, T., Kuper, F., Destexhe, E. andGarçon N.(2015): Non-clinical safety and biodistribution of AS03-adjuvanted inactivated pandemic influenza vaccines. J. Appl. Toxicol., 10:1002-1015.

36. Seubert, A., Calabro, S., Santini, L., Galli, B., Genovese, A. and Valentini S.(2011): Adjuvanticity of the oil-inwater emulsion MF59 is independent of Nlrp3 inflammasome but requires the adaptor protein MyD88. Proc. Natl. Acad. Sci. USA, 108: 1169-1174.

37. Szyszko, E., Brokstad, K. Cox, R. J. Hovden, Madhun, A. and Haaheim, L. R.(2006): Impact of influenza vaccine formulation with a detailed analysis of the cytokine response. Scandinavian Journal of Immunology, 64: $467-475$.

38. Viera, S.(2001): Adverse effects of adjuvants in vaccines. Whale, 8(2): 1-11.

39. Warwick, R. and Peter, W.(1973): Angiology. In: Gray's Anatomy. 35 ${ }^{\text {th }}$ ed., Longman,London,pp. 588-785. 